\title{
VALIDACIÓN DEL CUESTIONARIO EPIDEMIOLÓGICO EN SINTOMATOLOGÍA MENTAL -CESIM- EN POBLACIÓN ARGENTINA.
}

\author{
VALIDATION OF THE EPIDEMIOLOGICAL \\ QUESTIONNAIRE -CESIM- IN MENTAL \\ SYMPTOMATOLOGY IN ARGENTINIAN POP- \\ ULATION.
}

\section{Mariela Muñoz Rodríguez Celina Korzeniowski² \\ 'Lic. en Psicología. Becaria doctoral CONICET, Universidad Nacional de San Luis, Argentina Dra. en Psicología Investigadora Asistente INCIHUSA-CONICET Mendoza, Argentina.}

Trabajo recibido: 29 de diciembre 2018. Aprobado: 26 de abril

\section{VALIDAÇÃO DO QUESTIONÁRIO EPIDEMI- OLÓGICO NA SINTOMATOLOGIA MENTAL -CESIM- NA POPULAÇÃO ARGENTINA.}

\section{Resumen}

La planificación estratégica del sistema sanitario se basa en información epidemiológica. Estos datos se recaban principalmente de evaluaciones sobre las consultas que posee el sistema de salud y permiten estimar la población afectada. Las técnicas que se utilizan son principalmente la de organismos internacionales y estas no tienen consistencia ecológica ni están validadas en el país. Los objetivos de este estudio fueron analizar las propiedades psicométricas del Cuestionario Epidemiológico en Sintomatología Mental versión breve (CESIM) y brindar datos normativos para población adulta argentina. La investigación se enmarcó en un diseño instrumental. Participaron 1216 adultos de 18 a 90 años de edad, con una distribución proporcional en tres localidades argentinas: Gran Mendoza, La Plata y Avellaneda. A partir del estudio factorial del CESIM se obtuvo una estructura simple y clara del cuestionario compuesta por 37 ítems que se agruparon en 8 factores interpretables, los cuales explicaron el $46 \%$ de la varianza total del instrumento. El puntaje total del CESIM presentó una excelente consistencia interna. El estudio descriptivo de la sintomatología mental en las personas participantes indicó, en línea con investigaciones previas, que las mujeres y las personas con bajos niveles de instrucción 
presentaron mayores niveles de sintomatología mental. Los residentes de Mendoza presentaron los niveles más altos de sintomatología mental. Se concluye que el CESIM es un instrumento con adecuadas propiedades psicométricas y validez ecológica para evaluar la sintomatología mental en adultos argentinos.

Palabras clave: epidemiología, salud mental, técnicas psicológicas, adultos, Argentina.

\section{Abstract}

The strategic planning of the health system is based on epidemiological information. Data are mainly obtained from evaluations on consultations the health system has, and allow an estimation of the affected population. Techniques used mainly belong to international organisms and do not have ecological consistency and are not validated in the country. The objectives of this study were to analyze the short version of the Epidemiological Questionnaire on Mental Symptomatology (CESIM, for its Spanish acronym) and provide regulatory data for the adult population. Research presented an instrumental design. There were 1216 adults from 18 to 90 years old, with a proportional distribution in three Argentinian locations: Gran Mendoza, La Plata and Avellaneda. From the factorial analysis of CESIM, a simple and clear structure of the questionnaire was obtained, made up of 37 items grouped in 8 interpretable factors which explained $46 \%$ of the instrument total variance. CESIM total score presented excellent internal consistency. The descriptive study of mental symptomatology in the participants showed, in accordance with previous research, that women and people with low educational level presented higher levels of mental symptomatology. Mendoza residents presented the highest levels of mental symptomatology. We conclude that CESIM is an effective instrument to assess mental symptomatology in Argentinian adults since it presents appropriate psychometric properties and ecological validity.

Key words: epidemiology, mental health, psychological techniques, adults, Argentina.

\section{Resumo}

O planejamento estratégico do sistema de saúde é baseado em informações epidemiológicas. Esses dados são coletados principalmente a partir de avaliações sobre as consultas que o sistema de saúde possui e permitem estimar a população afetada. As técnicas utilizadas são principalmente aquelas de organizações internacionais e estas não possuem consistência ecológica nem são validadas no país. Os objetivos deste estudo foram analisar as propriedades psicométricas do Questionário Epidemiológico em Sintomatologia Mental versão curta (CESIM) e fornecer dados normativos para a população adulta argentina. A pesquisa foi enquadrada em um desenho instrumental. Participaram nela 1216 adultos com idades entre 18 e 90 anos de idade, com uma distribuição proporcional de três localidades argentinas: Gran Mendoza, La Plata e Avellaneda. Apartir do estudo fatorial de CESIM foi obtida uma estrutura simples e clara do questionário, composta de 37 itens que foram agrupados em 8 fatores interpretáveis que explicaram $46 \%$ da variância total do instrumento. A pontagem total do CESIM apresentou excelente consistência interna. $\mathrm{O}$ estudo descritivo da sintomatologia mental nos participantes indicou, em consonância com pesquisas anteriores, que mulheres e pessoas com baixa escolaridade apresentavam níveis mais elevados de sintomas mentais. Os moradores de Mendoza apresentaram os mais altos níveis de sintomas mentais. Conclui-se que o CESIM é um instrumento com adequadas propriedades psicométricas e validade ecológica para avaliar a sintomatologia mental em adultos argentinos.

Palavras chave: epidemiologia, saúde mental, técnicas psicológicas, adultos, Argentina. 
La planificación estratégica en salud para la organización del sistema sanitario contempla los procesos de salud-enfermedad-atención (S-E-A). Esta planificación se realiza principalmente a través de investigaciones epidemiológicas, cuyos datos provienen en su mayoría de las consultas realizadas por personas usuarias de las instituciones de salud(2.) De esta manera, se interpreta que las consultas realizadas en los efectores de salud dan cuenta de las necesidades presentes en la población y a partir de ellas se realizan estimaciones poblacionales. Sin embargo, diferentes características sociodemográficas y culturales afectan las variaciones entre quienes consultan y quienes presentan problemas relacionados con el aspecto mental de la salud. Argentina sólo posee un estudio poblacional probabilístico con población general, es decir que incluye diferentes miembros del país seleccionados de manera aleatoria y proporcional para representar la población argentina (3.) Si bien, las investigaciones epidemiológicas que responden a estudios de tamizaje no brindan un diagnóstico en profundidad(4.) permiten reconocer a personas con algún tipo de padecimiento y planificar no sólo sobre los datos de quienes consultan sino sobre el resto de la población. A su vez, repercuten en la posibilidad de realizar acciones de asistencia, rehabilitación, promoción y prevención de acuerdo a esas características.

La Atención Primaria de la Salud (APS) como estrategia principal del sistema de salud desde los acuerdos internacionales, marca necesidades de intervención, planificación y acompañamiento de acuerdo con las características del proceso de S-E-A de las poblaciones. Así, insta a que la planificación en salud sea tanto para aquellas personas sanas, quienes poseen alguna dolencia o malestar y aquellas que están enfermas(5.) Conocer las características del contexto, recursos y malestares permite poder adecuar los procesos de intervención a estos. Asimismo, la APS propone una participación plena de las comunidades, aspecto que sería necesario implementar para no sobre-asistir, sino desde su reconocimiento y participación, acompañarlas en sus procesos comunitarios de salud.

El siglo XX empieza a marcar avances en investigaciones en salud mental, reconociendo paulatinamente el sufrimiento de las personas con padecimiento mental. La OMS en 1946 define, en su carta fundacional, a la salud de un modo integral, es decir, como un estado de bienestar biológico, psicológico y social. Esto fortalece paulatinamente teorizaciones y prácticas que van incluyendo el aspecto mental de la salud como eje de indagación y atención(6.) Modificaciones en la manera de comprender los roles del estado en relación a la salud, experiencias como la de Trieste (Italia, liderada por Franco Basaglia), en relación a la atención de las personas con padecimiento mental, como desarrollos científicos, reclamos y propuestas de usuarios y familiares del sistema de salud mental, han hecho imprescindible revisar los modos de planificación de la política pública en torno al tema. A su vez, los cambios en los modos de vida han generado que, en América Latina y el Caribe, los trastornos mentales y neurológicos -medida en años de vida ajustados en función de la discapacidad (AVAD)- representen el 22,2\% de la carga total de enfermedad(7.) Estas cargas de padecimiento mental hacen necesario que se deban revisar las políticas públicas en torno a la salud mental. La OMS a través de la Declaración de Caracas (1990) insta a atender esta situación repercutiendo en Argentina con la sanción de la Ley Nacional de Salud Mental ( $\left.\mathrm{N}^{\circ} 26657,2010\right)$. A partir de estudios epidemiológicos realizados en Argentina con población general se conoce que un $14,8 \%$ de los habitantes posee en el transcurso del último año sintomatología que corresponde a un desorden del área mental, de los cuales el $25 \%$ posee un diagnóstico de desórdenes severos (presencia de trastorno bipolar, dependencia a sustancias, intento de suicidio, o puntuar por encima de 7 en escalas de discapacidad) y el 35,7\% moderado(3.) Sumado a esto, los datos del Instituto de evaluaciones y métricas para la salud (Institute for Health Metrics and Evaluations) sobre los problemas que al 2016 responden AVAD muestran que tres de ellos responden a problemas de salud mental: los trastornos depresivos y de ansiedad como segundas y quintas causas; y el 
asma, la décima(8.) A su vez, estudios que revisan datos epidemiológicos latinoamericanos identifican que la violencia está asociada a más de $10 \%$ de las causas de AVAD(9.)

A pesar de la magnitud de la carga que representan los trastornos mentales y neurológicos, la brecha del tratamiento es abrumadora, va desde $71,4 \%$ en los casos de consumo problemático de alcohol al 37,4\% en psicosis no afectivas(10.) El 11,6\% de la población argentina ha consultado en el último año servicios de salud mental, sin embargo,solo el $30,2 \%$ de las personas con sintomatología severa lo hicieron(3.) Estos datos muestran que los desafíos que tenemos en relación a la atención y acompañamiento a personas con padecimiento mental son muy grandes en nuestro país. La sanción de la ley no sólo muestra la importancia de abordar la problemática sino que evidencia y redefine paradigmas para su abordaje y fija desafíos. La perspectiva desde donde se debe trabajar en salud mental es el paradigma de derechos y se basa en la APS para su abordaje; por lo tanto, en el trabajo con las comunidades donde las personas con padecimiento mental viven(5.)

En un trabajo intersectorial la Dirección de Salud Mental y Adicciones de la Nación junto a usuarios, familiares e instituciones afines a la temática realizan el Plan Nacional de Salud Mental(11.) En él se evidencian distintas problemáticas en torno al área desalud mental y una de ellas se refiere a la necesidad de desarrollar estudios epidemiológicos sobre en el país sobre la temática. Esto debido a que Argentina cuenta con escasos datos epidemiológicos en relación al tema y los que posee son sobre usuarios y no de población general, salvo el estudio realizado por Stagnaro y colaboradores (2018), el estudio anterior es de 1979(3,10.) Las investigaciones están basadas en categorías de enfermedad mental y se realizan a partir de instrumentos traducidos y validados o bien propuestos por instituciones internacionales. Estos cuentan con el riesgo de dar lugar a interpretaciones erróneas debido a diferencias culturales y de lenguaje, como por la posibilidad trasponer valoraciones a distintos contextos sin validaciones.

A continuación se mencionan brevemente algunos de los cuestionarios más utilizados para realizar investigaciones en Argentina. Uno de ellos es el Listado de Síntomas 90 (SCL 90, por sus siglas en inglés: Symptom Check List 90) de Derogatis, Lipman y Covi (1973) en Salud Mental validado en Argentina(12.) El Kessler Psychological Distress Scale (K-10) está validado en Argentina(13), evalúa síntomas vinculados a la depresión y la ansiedad. Otro bastante utilizado es el Inventario Clínico Multiaxial de Millon-II (MCMI-II), que mide síntomas vinculados con trastornos de la personalidad y dificultades emocionales e interpersonales (14.)

En el orden de las baterías, existen a nivel internacional distintos conjuntos de instrumentos diseñados por organismos internacionales sin ser validados en Argentina. El Composite International Diagnostic Interview (CIDI15), es una Entrevista Diagnóstica Internacional que permite medir la prevalencia de los trastornos mentales, su gravedad, determinar la carga de estos trastornos, evaluar el uso de servicios, uso de medicamentos y a quién se trata. El Structured clinical interview for DSM-V - (SCID- APA16) es la última versión de una técnica elaborada en base al manual diagnóstico de salud mental. Posee diferentes versiones: para clínica, para investigaciones y para ensayos clínicos. El Cuestionario para la Evaluación Clínica en Neuropsiquiatría (SCAN17) es un conjunto de instrumentos destinados a analizar, medir y clasificar la psicopatología y la conducta asociada a las principales alteraciones psiquiátricas de la edad adulta. La Present State Examination (PSE17) compone la SCAN y posee dos grandes ejes para el diagnóstico: uno referido a síntomas físicos, depresivos y ansiedad, y otro, relacionado con problemas psicóticos y cognitivos. En este trabajo se busca analizar las propiedades psicométricas del Cuestionario Epidemiológico en Sintomatología Mental -CESIM. El instrumento fue diseñado en la provincia de Córdoba, Argentina por la Dirección General de Salud Mental, con la colaboración de las instituciones SECYTECOR y CONICOR y la participación de profesores, en ese momento, de la Escuela de Psicología de la Universidad Nacional de Córdoba. En el manual de la técnica se presenta la versión original y su versión breve construida luego del 
análisis factorial exploratorio (AFE)(1.) El CESIM fue elaborado con fines epidemiológicos $y$, en consecuencia, resulta en un instrumento de tamizaje o screening, que brinda una medida rápida y global de sintomatología mental.

Si bien la técnica permite evaluar la severidad de la sintomatología mental, diferentes estudios que la han utilizado en articulación con preguntas de identificación de problemas de tipo emocional, indican alta correlación con el uso de servicios de salud mental 2,18,19. El malestar subjetivo se refiere al sufrimiento, descontento o sensación desagradable que un individuo experimenta en un momento determinado, independientemente de cómo lo exprese(20.) Así, la sintomatología ha aparecido relacionada a la autopercepción de malestar como a consultas con los y las profesionales de la salud,llevando a las personas a solicitar asistencia clínica.

Una de las dificultades del CESIM radica en que presenta una estructura factorial compleja $(1,21,22,23$. $)$ En su versión extensa, compuesta por 78 ítems, se extrajeron 22 factores que explicaban el $60 \%$ de la varianza, aunque los autores recomendaron retener aquellos factores que presentaban un agrupamiento de ítems coherente e interpretable desde la teoría. Si bien, postulan retener entre 5 y 7 factores que explican entre el $31 \%$ y el $35.5 \%$ de la varianza, sugieren interpretar el puntaje global arrojado por el cuestionario y atender a cuatro de las dimensiones de la escala: angustia/ansiedad/temores, relaciones con el propio cuerpo, contacto con la realidad y autoestima/inseguridad(1.)

En base a estos resultados, se propuso una versión abreviada del CESIM 1.2 compuesta por 54 ítems1, en la cual se retuvieron los mejores 49 ítems de la versión original y se incorporaron 6 ítems nuevos, tres para valorar depresión y tres para evaluar conductas obsesivo-compulsivas. En esta versión, el CESIM quedó constituido por un gran eje de conductas y cogniciones asociadas al malestar subjetivo (46 ítems) y otra que corresponde a síntomas de índole psicosomático ( 8 ítems). Los autores y autora no informan estudios de la estructura factorial de esta versión, por lo cual calculan la sintomatología mental sin incluir los ítems nuevos. El puntaje global del CESIM 1.2 presentó un adecuado valor de confiabilidad test-retest $(\mathrm{r}=.86)$ para la muestra de Córdoba y Oliva1.

Se han informado niveles de sintomatología mental en personas mayores de 18 años argentinas empleando la versión breve del CESIM, sin embargo, los mismos son aproximados debido a que se construyen en base a la depuración de la técnica, pero sin agregar la evaluación de los nuevos ítems en los análisis. A partir de esta aproximación los autores generan una propuesta de interpretación escalar de los datos con percentiles (10 y 90) y cuartiles $(25,50$ y 75$)$.

En Argentina el CESIM se ha utilizado en diferentes estudios. En 1995 se lo utilizó en una investigación comparativa de sintomatología mental entre personas afectadas por la desocupación y ocupadas utilizando el puntaje total de sintomatología como eje de análisis(21.) En relación al eje de trabajo, se realizó un estudio descriptivo ítem por ítem de los síntomas mentalesen docentes en escuelas de Córdoba(22.) También, en la provincia de Córdoba han utilizado la técnica y los valores propuestos en el manual del CESIM para establecer los criterios de inclusión y exclusión de participantes en un estudio de comorbilidad depresiva en mujeres con trastornos de conducta alimentaria(24.) En un estudio comparativo entre las localidades de La Plata y Avellaneda se propuso analizar los síntomas en cuatro niveles: bajo, medio, alto y muy alto19. En estudios realizados en la provincia de Mendoza, se utilizaron dichos niveles de malestar a fin de describir malestar en diferentes muestras de la provincia $(2,14,20$.) Finalmente, en Córdoba en un estudio de ansiedad ante los exámenes, procrastinación y síntomas mentales en estudiantes, debido a la falta de datos sobre la estructura interna del CESIM, los autores realizan un AFE para la muestra en estudio(23.) Los datos obtenidos fueron utilizados sólo para sustentar empíricamente el agrupamiento de los ítems del cuestionario en los tres factores que informan. En suma, los estudios presentados indican que el CESIM es un instrumento ampliamente usado para valorar sintomatología mental en nuestro país, aunque presenta algunas difi- 
cultades psicométricas, especialmente una estructura factorial compleja. Este aspecto hace necesario realizar nuevos estudios del instrumento.

La presente investigación se propone como objetivos: 1) analizar las propiedades psicométricas del CESIM versión breve y 2) brindar datos normativos de sintomatología mentalen personas adultas argentinas según características sociodemográficas.

\section{Método}

Este estudio se enmarca en un diseño instrumental que corresponde a aquellos centrados en la adaptación de pruebas o en la medición de características psicómetricas de instrumentos (25.)

Para realizar dicha investigación se utilizaron los datos correspondientes a dos estudios realizados en el país entre los años 2011-2014.

\section{Muestra}

Se empleó una muestra probabilística y representativa de tres regiones de Argentina. La muestra total de la presente investigación estuvo conformada por 1216 personas adultas de ambos sexos, de 18 a 90 años de edad, radicados en Argentina. Los participantes son 663 mujeres (m) y 553 varones (v). Las personas pertenecen a tres ciudades del país: Avellaneda 401 (v: 188 y m: 213) y La Plata 400 (v: 176 y m: 224) de la provincia de Buenos Aires, y Guaymallén 415 (v: 189 y m: 226) de la provincia de Mendoza.

La muestra total seleccionada fue probabilística y al azar, por lo cual se mantuvieron en cada zona la proporcionalidad de cuota según edad y sexo,de acuerdo al Censo Nacional de Población, Hogares y Viviendas 200126.Para la selección de la muestra se sortearon en los tres lugares de pesquisa 50 manzanas para la recolección de datos.En la conformación de la muestra se tuvieron en cuenta la proporción de sexo y siete rangos etarios: de 18 a 24;de 25 a 34; de 35 a 44; de 45 a 54; de 55 a 64; de 65 a 74; de 74 en adelante.

Una vez conformada la muestra total, se realizó una división de la misma en dos submuestras con una selección proporcional y aleatoria. De esta manera, se conformó una submuestra para realizar el estudio instrumental y otra, para brindar datos normativos de sintomatología mental para personas adultas argentinas. La primerasubmuestra quedó constituida por 406 personas adultas: 134 de Avellaneda (71 mujeres), 135 de La Plata (75 mujeres) y 137 de Mendoza (74 mujeres). La segunda submuestra estuvo conformada por 810personas adultas:267 de Avellaneda (142 mujeres), 265 de La Plata (149 mujeres) y 278 de Mendoza (152 mujeres).

\section{Instrumento}

Cuestionario Epidemiológico de Sintomatología Mental versión breve1(CESIM): es un instrumento de tamizaje que valora sintomatología mental autopercibida. Fue diseñado y construido en Córdoba, Argentina, en el año 1993 por un equipo de investigadores e investigadoras de la Dirección General de Salud Mental de dicha Provincia.Este instrumento ha sido validado y sometido a estudios de confiabilidad1. Además, la validez ecológica, satisfechas las exigencias psicométricas, deviene del hecho de haber sido construido dentro del mismo país.

El CESIM versión breve está compuesto por 54 ítems. Es un cuestionario de autoinforme, en el cual se emplea una escala likert de 4 opciones para brindar la respuesta: si, no,a veces, no sabe/no contesta. En el presente estudio se consideraron tres opciones de respuestas, tratando como valor perdido la opción no sabe/no contesta. A diferencia de la versión original las puntuaciones de los síntomas psicosomáticos también fueron evaluadas con una escala likert de tres puntos: si, no y a veces.

\footnotetext{
${ }^{3}$ Uno realizado en 2011 desde la Universidad de Buenos Aires, dirigido por el Dr. Enrique Saforcada“Estudio epidemiológico comparativo de sintomatología de salud mental en adultos en el partido de Avellaneda y la Ciudad de la Plata" y otro realizado en 2014 desde la Universidad de Mendoza, dirigido por Mariela Muñoz "Estudio Epidemiológico comparativo de sintomatología de Salud Mental en adultos en comunidades urbana y rural de la Provincia de Mendoza".
} 


\section{Procedimiento}

Se capacitó a equipos de encuestadores y encuestadoras para el estudio tanto en la selección de los y las participantes como en la técnica y modalidad de toma. Se realizaron las tomas de los cuestionarios casa por casa.Para la recolección de datos se trabajó a partir de los puntos muestrales previamente seleccionados y se recorrieron lascuadras desde la vereda norte en el sentido de las agujas del reloj. Se asignaron cuotas a los y las entrevistadoras según sexo y edad. Luego de iniciada la entrevista y de recolectar los datos sociodemográficos de composición del hogar se procedió a seleccionar al componente de acuerdo a las personas presentes en el momento de la visita que accedieran a la realización de la entrevista y que cumplieran con las cuotas asignadas hasta completar las proporciones por sexo y edad en cada una de las zonas de trabajo.

\section{Análisis de datos}

Se empleó el paquete estadísticos SPSS .23 (StatisticalPackageforthe Social Sciences Inc) para el análisis de los datos.

En primer lugar, se prepararon los datos, las respuestas no sabe/no contesta, se consideraron como valores perdidos. Se empleó el análisis de valores perdidos del programa SPSS versión 23 para identificar el porcentaje de casos perdidos, ninguno de los ítems superó el valor del 5\%.Los valores perdidos fueron imputados por la moda.

Para dar respuesta al primer objetivo del trabajo, se anailzaron las propiedades psicométricas del CESIM versión breve. Para tal fin, se realizóun análisis factorial exploratorio (AFE) del instrumento en una muestra de 406 personas adultas. Se calculó la medida de adecuación muestral de Kaiser Mayer y Olkin (KMO) y la prueba de esfericidad de Bartlett. Para facilitar la interpretación de la solución factorial se empleó la rotación oblicua Promax. Posteriormente, se analizó la consistencia interna de las ochofactores y del factor general de sintomatología mental que arroja el CESIM, lo que se complementó con un análisis de discriminación de los ítems. La consistencia interna se midió con el estadístico alfa de Cronbach y se empleó el estadístico $r$ de Pearson para medir la magnitud de la correlación ítem- resto del test.

En línea con el segundo objetivo del trabajo, se calcularon los estadísticos descriptivos y de posición para la medida global de sintomatología mental y de los ocho factores del CESIM para personas adultas argentinas.Asimismo, se realizaron estudios comparativos a fin de valorar si la sintomatología mental fluctuaba según el sexo, la edad, el lugar de residencia, el nivel educativo y el estado civil de los participantes. Para realizar el mencionado estudio se emplearon tablas de contingencia y el estadístico de Chi-cuadrado con un nivel de significancia de .05 .

\section{Consideraciones éticas}

A todas las personas encuestadas les fue solicitado consentimiento informado, asegurando anonimato y reserva de la identidad. Asimismo, se les manifestó que su participación era voluntaria y que podía declinarla en el momento que así lo consideraran.

\section{Resultados}

\section{Estudio instrumental}

\section{Análisis Factorial Exploratorio}

Se estudió la estructura factorial de la versión breve del CESIM (versión original) compuesta por 54 ítems en una muestra de personas adultas argentinas. Dicha versión posee 8 ítems de enfermedades psicosomáticas y 46 ítems que se refieren a síntomas de predominio mental. Se realizaron varios análisis factoriales exploratorios con el método de componentes principales y con rotación oblicua. 
Como primera dificultad, se observó que los ocho ítems de las enfermedades psicosomáticas presentaban agrupamientos con uno o dos ítems conformando factores poco coherentes y no interpretables desde la teoría. Atendiendo a que estas dificultades han sido informadas en estudio previos del instrumento $(1,21,27$,) se decidió realizar un nuevo AFE sin incluir estos indicadores.

Se realizó un AFE con el pool de 46 ítems del CESIM 1.2. Se obtuvo un índice de KMO de .90 y X2 $(1035)$ de Bartletett $=5129.26(\mathrm{p}<.001)$. El estudio inicial arrojó 13 factores que explicaban el 58\% de la varianza. Tras depurar la matriz factorial, descartando aquellos ítems con baja comunalidad, alta complejidad factorial o bajo peso en el factor que se pretendía operacionalizar, se llegó a una estructura clara y compuesta por 37 ítems que pesaron en ocho factores:a) Angustia, b) Agresividad/autoestima, c) Ansiedad, d) Ideas de persecución, e) Inseguridad/dependencia, f) Vivencias de malestar relacionadas con el cuerpo, g) Alucinaciones-delirios, h) Desorientación/pérdida de memoria. Estos 8 factores explicaron el $46 \%$ de la varianza total.

La Tabla 1 presenta la estructura factorial del CESIM conformada por 37 ítems e indica que los pesos factoriales para el factor Angustia oscilaron entre .33 a .69; para el factor Agresividad/autoestima entre .31y .76; para el factor Ansiedad entre .75 y .82; para el factor Ideas de persecución entre .51 y .93; para el factor Inseguridad/independencia con el cuerpo entre .38 y .79; para el factor Vivencias de malestar relacionadas entre .38 y .86 , para el factor Alucinaciones/delirios entre .46 y .78, y para el factor Desorientación/ pérdida de memoria entre 46 y .77.

Como puede apreciarsela mayoría de los indicadores presentaron saturaciones mayores a .40 en los subfactores (ver Tabla 1). Si bien, se observó que cuatro ítems presentaron moderada complejidad factorial: el ítem 19: "Me pasa que no sé en qué día ni en qué mes estoy", ítem 24 "Me miro al espejo y no me reconozco mí mismo", ítem 26 "Me sucede que objetos o sombras que veo creo que son personas", ítem 27 "Cosas que otros no toman a mal a mí me provocan bronca", se decidió conservar dichos reactivos debido a que alcanzaron una saturación superior a .40 en el factor interpretable.Sin embargo, se surgiere en estudio posteriores atender al comportamiento de estos indicadores(ver Tabla 1).

\section{Consistencia interna y análisis de los ítems}

En la segunda fase del estudio se exploró la consistencia interna de las ocho subescalas del CESIM y del puntaje global de la escala, lo que se complementó, con el análisis de discriminación de los ítems. La consistencia interna del puntaje global del CESIM compuesto por los 37 ítems (en adelante, CESIM 37)fue de $\alpha=.91$.

Con relación a los factores extraídos, se apreció que elFactor 1: Angustia, compuesto por 7 ítems, presentó una consistencia interna de $\alpha=.78$, el Factor 2: Agresividad /autoestima, compuesto por 8 ítems, presentó un consistencia interna de $\alpha=.76$, el Factor 3: Ansiedad, conformado por 3 indicadores, presentó una consistencia de $\alpha=.72$, el Factor 4: Ideas de persecución, compuesto por 5 ítems, presentó un coeficiente alfa de $\alpha=.67$, el Factor 5: Inseguridad/dependencia, compuesto por 5 ítems, presentó una consistencia de $\alpha=.66$, el Factor 6: Vivencias de malestar relacionadas con el cuerpo, compuesto por 3 ítems, presentó un coeficiente alfa de .60, el Factor 7: Alucinaciones/delirios, compuesto por tres ítems, presentó una confiablidad de $\alpha=.49$ y el Factor 8: Desorientación/alteraciones de memoria, compuesto por 3 ítems, presentó una confiabilidad de $\alpha=.68$ (ver Tabla 1). El análisis de discriminación de los ítems permitió observar correlaciones ítem-dominio de bajas a moderadas para el CESIM 37. De este análisis se observaron correlaciones entre .43 a .63 entre los ítems del factor Angustia, entre .36 a .55 entre los ítems del factor Agresividad/autoestima, entre .51 a .56 entre los indicadores del factor Ansiedad, entre .35 a .52 entre los ítems del factor Ideas de persecución, entre .36 a .50 entre los ítems del factor Inseguridad/dependencia, entre .38 a .44 entre los ítems de factor Vivencias 
relacionadas con el cuerpo, entre .29 y .35 entre los indicadores del factor Alucinacionesdelirios, y entre .36 a .42 en el factor Desorientación/alteraciones de memoria. Asimismo, se analizaron las correlaciones ítem-resto del test, las cuales oscilaron entre .29 a .65 para el CESIM 37.

Atendiendo a lo postulado por los autores del instrumento quienes consideran de importancia incluir los indicadores de enfermedades psicosomáticas dentro del constructo de síntomas afectivos, a pesar de que no puedan agruparse en subfactores interpretables1, se decidió calcular la consistencia interna del CESIM incluyendo estos ocho indicadores. Los resultados arrojaron un valor de alpha de .91 para el pool de 45 ítems (en adelante, CESIM 45), vale decir para los 37 ítems previamente analizados más los 8 reactivos de enfermedades psicosomáticas. Finalmente,se calcularon las correlaciones ítems-resto del test para el CESIM 45 obteniendo valores que oscilaron entre .07 a .65 . El propósito depresentar los valores de consistencia interna y de discriminación ítems-resto del test para el CESIM 45 se fundamenta en que estudios posteriores puedan disponer de estos datos a fin de continuar indagando las propiedades psicométricas de este instrumento.

Tabla $\mathbf{N}^{0}$ 1: Estructura factorial del CESIM versión breve rotada (Promax) y consistencia interna.

\begin{tabular}{|c|c|c|c|c|c|c|c|c|}
\hline Item & F1 & F2 & F3 & $\mathrm{F} 4$ & F5 & F6 & F7 & F8 \\
\hline 1. Me asusto sin ningún motivo. & .51 & & & & & & & \\
\hline $\begin{array}{l}\text { 2. Estoy tan triste y preocupado/a que siento una opresión en } \\
\text { el pecho que me ahoga. } \\
\text { 3. Siento que tengo un nudo en la garganta. }\end{array}$ & .47 & & & & & & & \\
\hline 4. Para mí la mejor solución sería morir. & .33 & & & & & & & \\
\hline 5. Tengo pesadillas. & .68 & & & & & & & \\
\hline 6. He perdido el apetito. & .69 & & & & & & & \\
\hline 7. Siento mucho cansancio y ganas de dormir todo el día. & .69 & & & & & & & \\
\hline 8. Siento que tengo muchos y muy graves defectos. & & .48 & & & & & & \\
\hline $\begin{array}{l}\text { 9. Agredo a los/as demás y no me doy cuenta hasta que me } \\
\text { lo dicen. }\end{array}$ & & .76 & & & & & & \\
\hline 10. Pienso que en general las reglas no son válidas para mí. & & .45 & & & & & & \\
\hline $\begin{array}{l}\text { 11. Me siento más molesto/a que la mayoría de la gente si no } \\
\text { me encuentro atractivo. }\end{array}$ & & .31 & & & & & & \\
\hline 12. Cuando me peleo o discuto con alguien pierdo el control. & & .32 & & & & & & \\
\hline 13. Discuto y peleo mucho. & & .73 & & & & & & \\
\hline $\begin{array}{l}\text { 14. Cosas que otros no toman a mal a mí me provocan } \\
\text { bronca. }\end{array}$ & & .41 & & & & & & \\
\hline 15. Detalles insignificantes me provocan una gran irritación. & & .55 & & & & & & \\
\hline 16. Estoy inquieto/a y nervioso/a. & & & .82 & & & & & \\
\hline 17. Estoy ansiosa/o. & & & .80 & & & & & \\
\hline 18. Estoy tensa/o. & & & .75 & & & & & \\
\hline $\begin{array}{l}\text { 19. Las/os demás me molestan, me persiguen, no me dejan } \\
\text { progresar. }\end{array}$ & & & & .93 & & & & \\
\hline 20. Mis pensamientos son dominados por fuerzas extrañas. & & & & .51 & & & & \\
\hline $\begin{array}{l}\text { 21. Descubro doble sentido o amenazas encubiertas en lo que } \\
\text { la gente dice o hace. } \\
\text { 22. Me miro al espejo y no me reconozco a mí mismo/a. }\end{array}$ & & & & .56 & & & & \\
\hline $\begin{array}{l}\text { 23. Pienso que hay personas que para que me vaya mal me } \\
\text { ponen obstáculos. }\end{array}$ & & & & .48 & & & & \\
\hline $\begin{array}{l}\text { 24. Necesito mucho apoyo de otras/os en forma de consejo o } \\
\text { estímulo antes de tomar decisiones cotidianas. }\end{array}$ & & & & & .44 & & & \\
\hline 25. No puedo tomar decisiones. & & & & & .79 & & & \\
\hline
\end{tabular}


26. No puedo emprender ninguna actividad sin que me ayuden.

27. Me cuesta hacer las cosas debido a que no puedo determinar cómo empezar o enfrentar un problema.

28. Me siento inferior a los otros.

29. Siento que me pasan cosas nuevas en mi cuerpo que no se explicar.

30. Sufro de malestares físicos que los médicos no saben a qué se deben.

31. Me siento enfermo/a

32. Me sucede que objetos o sombras que veo creo que son personas.

33. Suelo escuchar cosas que otras/os no escuchan.

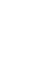

.75

34. Me sucede que sonidos que escucho creo que son voces.

35. Me pasa que no sé en qué día ni en qué mes estoy.

36. Tengo dificultades para recordar las cosas.

37. Salgo a hacer algo y después no me acuerdo qué era.

.40

.38

.38

.86

.78

.46

.78

.69

$\begin{array}{llllllll}.78 & .76 & .72 & .67 & .66 & .60 & .49 & .58\end{array}$

\title{
Estudio descriptivo
}

Se estimaron los estadísticos descriptivos y de posición a fin de conocer la sintomatología mental de acuerdo a los factores sociodemográficos en la muestra en estudio. Se calcularon los percentiles para identificar los puntos de corte a fin de elaborar las categorías: bajo, medio, alto y muy alta sintomatología mental (ver Tabla 2).

Tabla $N^{0}$ 2: Estadísticos descriptivos para sintomatología mental y los ocho factores que conforman el CESIM en una muestra de adultos y adultas argentinas

\begin{tabular}{llllllll}
\hline Variables & $M$ & $D E$ & Rango & 25 & 50 & 75 & 90 \\
\hline Angustia & 2,34 & 2,67 & $0-14$ &, 00 & 2,00 & 4,00 & 6,00 \\
Agresividad & 3,15 & 3,10 & $0-16$ & 1,00 & 2,00 & 5,00 & 7,00 \\
Ansiedad & 2,20 & 1,92 & $0-6$ &, 00 & 2,00 & 4,00 & 5,00 \\
Persecución & 1,26 & 1,79 & $0-10$ &, 00 &, 00 & 2,00 & 4,00 \\
Inseguridad & 1,55 & 1,87 & $0-10$ &, 00 & 1,00 & 2,00 & 4,00 \\
Hipocondría &, 79 & 1,36 & $0-6$ &, 00 &, 00 & 1,00 & 2,00 \\
Delirios &, 66 & 1,24 & $0-6$ &, 00 &, 00 & 1,00 & 2,00 \\
Desorientación & 1,33 & 1,56 & $0-6$ &, 00 & 1,00 & 2,00 & 4,00 \\
CESIM 37 & 13,29 & 10,88 & $0-74$ & 5,00 & 11,00 & 19,00 & 29,00 \\
Psicosomáticos & 2,42 & 2,54 & $0-14$ &, 00 & 2,00 & 4,00 & 6,00 \\
CESIM 45 & 15,72 & 12,03 & $0-90$ & 7,00 & 13,00 & 22,00 & 32,00 \\
\hline
\end{tabular}

$\mathrm{n}=810$. Nota: CESIM 37 refiere al puntaje total del cuestionario sin incluir los indicadores de enfermedades psicosomáticas. CESIM 45 refiere al puntaje total del cuestionario incluyendo los indicadores de enfermedades psicosomáticas.

Las autoras y autores del CESIM (1) proponen cuatro categorías de análisis de las puntuaciones: bajo, medio, alto y muy alto. De acuerdo con estos, se estimó el porcentaje de personas que presentaron baja (primer cuartil), media (segundo cuartil), alta (tercer cuartil hasta el percentil 90) y muy altasintomatología mental(percentil 90) empleando el puntaje total del cuestionario que incluye las enfermedades psicosomáticas (CESIM 45) (ver Tabla 3). Posteriormente, se realiza un análisis descriptivo en función de variables sociodemográficas: edad, sexo, nivel de educación, estado civil y región geográfica. 
Tabla $\mathbf{N}^{0}$ 3: Nivel de sintomatología mental según edad de las personas participantes $(\mathrm{n}=810)$

\begin{tabular}{lllll}
\hline \multirow{2}{*}{ Edad } & \multicolumn{4}{c}{ CESIM 45 } \\
\cline { 2 - 5 } & Baja & Media & Alta & Muy Alta \\
\hline $18-24$ & $11,3 \%$ & $18,9 \%$ & $25,8 \%$ & $18,2 \%$ \\
$25-34$ & $20,3 \%$ & $21,8 \%$ & $20,8 \%$ & $21,6 \%$ \\
$35-44$ & $19,4 \%$ & $14,2 \%$ & $14,2 \%$ & $17 \%$ \\
$45-54$ & $14 \%$ & $11,8 \%$ & $14,2 \%$ & $14,8 \%$ \\
$55-64$ & $15,3 \%$ & $15 \%$ & $11,7 \%$ & $13,6 \%$ \\
$65-74$ & $13,1 \%$ & $11 \%$ & $9,2 \%$ & $8 \%$ \\
75 o más & $6,8 \%$ & $7,3 \%$ & $4,2 \%$ & $6,8 \%$ \\
\hline
\end{tabular}

Nota: $\mathrm{X} 2(18)=17,97, \mathrm{p}=.457$

La distribución de sintomatología mental en función de la edad de las personas participantes no registró diferencias significativas $(\mathrm{X} 2(18)=17,97, \mathrm{p}=.457)$.

Tabla $\mathbf{N}^{0}$ 4: Nivel de sintomatología mental según sexo de las personas participantes $(\mathrm{n}=810)$

\begin{tabular}{lllll}
\hline \multirow{2}{*}{ Sexo } & \multicolumn{4}{c}{ CESIM 45 } \\
\cline { 2 - 5 } & Bajo & Medio & Alto & Muy Alto \\
\hline Varón & $51,8 \%$ & $44,6 \%$ & $45,8 \%$ & $31,8 \%$ \\
Mujer & $48,2 \%$ & $55,4 \%$ & $54,2 \%$ & $68,2 \%$ \\
\hline \multicolumn{5}{c}{ Nota: X2(3)= $10,32, \mathrm{p}=.016$}
\end{tabular}

Se registraron diferencias en la distribución de los niveles de sintomatología mental en función del sexo de las personas participantes $(\mathrm{X} 2(3)=10,32, \mathrm{p}=.016)$, observándose que las mujeres presentaron porcentajes más elevados en malestar alto $(54,2 \%)$ y muy alto $(68,2 \%)$ en comparación con los varones (alto:45,8\%, muy alto: $31,8 \%)$ (Tabla 4).

Tabla $\mathbf{N}^{\mathbf{0}}$ 5: Nivel de sintomatología mental según nivel educativo de las personas participantes $(\mathrm{n}=810)$

\begin{tabular}{lllll}
\hline Nivel Instrucción & \multicolumn{3}{c}{ CESIM 45 } \\
\cline { 2 - 5 } & Bajo & Medio & Alto & Muy Alto \\
\hline Sin escolaridad/Primaria & $5,9 \%$ & $8,1 \%$ & $9,2 \%$ & $19,5 \%$ \\
incompleta & & & & \\
Primaria completa & $14,9 \%$ & $19,9 \%$ & $24,2 \%$ & $23 \%$ \\
Secundaria incompleta & $11,7 \%$ & $13,4 \%$ & $25 \%$ & $21,8 \%$ \\
Secundaria completa & $23,4 \%$ & $24,9 \%$ & $21,7 \%$ & $21,8 \%$ \\
Terciaria incompleta & $6,8 \%$ & $8,9 \%$ & $6,7 \%$ & $5,7 \%$ \\
Terciaria completa & $8,1 \%$ & $6 \%$ & $4,2 \%$ & $3,4 \%$ \\
Universitaria incompleta & $14 \%$ & $11 \%$ & $8,3 \%$ & $3,4 \%$ \\
Universitaria completa & $15,3 \%$ & $7,6 \%$ &, $8 \%$ & $1,1 \%$ \\
\hline
\end{tabular}

Se registraron diferencias en la distribución de los niveles de sintomatología mental según el nivel de escolaridad de las personas participantes $(\mathrm{X} 2(24)=73,56, \mathrm{p}<.001)$, observándose que los mayores niveles de malestar se registraron en quienes alcanzaron un bajo nivel de instrucción (sin escolaridad a secundaria incompleta) (Tabla 5). En cambio, en los niveles de instrucción superior (terciario y de grado completo o incompleto), los niveles altos y muy altos de malestar concentran entre el 1\% y el 8,3\% de carga de malestar. 
Tabla $\mathbf{N}^{\mathbf{0}}$ 6: Nivel de sintomatología mental según estado civil de las personas participantes $(\mathrm{n}=810)$

\begin{tabular}{lllll}
\hline & \multicolumn{4}{c}{ CESIM 45 } \\
\cline { 2 - 5 } Estado civil & Bajo & Medio & Alto & Muy Alto \\
\hline Soltero/a & $30,2 \%$ & $31 \%$ & $34,2 \%$ & $26,4 \%$ \\
Casado/a & $40,5 \%$ & $38,6 \%$ & $22,5 \%$ & $36,8 \%$ \\
Viudo/a & $8,6 \%$ & $9,4 \%$ & $7,5 \%$ & $4,6 \%$ \\
Separado/a & $2,7 \%$ & $3,7 \%$ & $11,7 \%$ & $8 \%$ \\
En pareja & $13,1 \%$ & $14,4 \%$ & $22,5 \%$ & $23 \%$ \\
Divorciado/a & $5 \%$ & $2,9 \%$ & $1,7 \%$ & $1,1 \%$ \\
\hline
\end{tabular}

Nota: $\mathrm{X} 2(15)=38,68, \mathrm{p}=.001$

La prevalencia de sintomatología mental varió en función del estado civil de las personas participantes $(\mathrm{X} 2(15)=38,68, \mathrm{p}=.001)($ Tabla 6$)$. Las personas solteras presentaron mayor porcentaje de sintomatología mental alto $(34,2 \%)$ y las personas casadas registraron el porcentaje más elevado en sintomatología mentalmuy alto $(36,8 \%)$. En consecuencia, podría afirmarse que estos dos grupos presentaron los mayores niveles de malestar, seguidos por el grupo en pareja. Los grupos restantes presentaron menores niveles de malestar alto y muy alto los cuales oscilaron entre el $1,7 \%$ y el $11,7 \%$, y entre el $1,1 \%$ y el $4,6 \%$, respectivamente. Resulta importante mencionar que en estos grupos se concentra el porcentaje más alto de la población.

Tabla $\mathbf{N}^{0}$ 7: Nivel de sintomatología mental según localidad de las personas participantes $(\mathrm{n}=810)$

\begin{tabular}{|c|c|c|c|c|}
\hline \multirow[b]{2}{*}{ Localidad } & \multicolumn{4}{|c|}{ CESIM 45} \\
\hline & Bajo & Medio & Alto & Muy Alto \\
\hline Avellaneda & $33,8 \%$ & $34,4 \%$ & $32,5 \%$ & $25 \%$ \\
\hline La Plata & $45,9 \%$ & $33,3 \%$ & $19,2 \%$ & $14,8 \%$ \\
\hline Mendoza & $20,3 \%$ & $32,3 \%$ & $48,3 \%$ & $60,2 \%$ \\
\hline
\end{tabular}

Finalmente, se observó que la prevalencia de sintomatología mental varió en función de la localidad de residencia de las personas participantes $(\mathrm{X} 2(6)=66,54, \mathrm{p}=<.001)$ (tabla 7). Mendoza presentó los niveles más altos de malestar, registrándose que el 48,3\% de las personas encuestadas exhibió altos niveles de malestar y el 60,2\% niveles muy altos de malestar. En segundo lugar, se ubicó la localidad de Avellaneda de la provincia de Buenos Aires, en la cual se observó que el 32,5\% de las personas participantes presentó niveles altos de malestar y el 25\% niveles muy altos. En la localidad de La Plata, provincia de Buenos Aires, la mayoría de las personas presentaron niveles bajos $(45,9 \%)$ o medios $(33,3 \%)$ de sintomatología mental.

\section{Discusion}

El estudio instrumental de CESIM versión breve, permitió identificar una estructura simple, coherente e interpretable, compuesta por 37 ítems agrupables en 8 factores que explican el $46 \%$ total de la varianza del instrumento. En relación con los factores extraídos, se observó que tres presentaron adecuados valores de confiabilidad (superiores a $\alpha=.70$ ), otros dos presentaron valores aceptables $(\alpha=.70)$ y los tres restantes presentaron bajos valores de confiabilidad (menores a $\alpha=.60$ ). La consistencia interna del puntaje total de la técnica resultó excelente.

Los ocho factores hallados presentan similitud con estudios previos, así comotambién algunas disidencias. Se halló un factor no sometido a pruebas previamente, el factor 
Angustia, el cual presentó alta consistencia interna. A su vez, se observan dos factores que agrupan dos aspectos sintomáticos que generalmente se evalúan separados. Uno de ellos, es Agresividad/autoestima. Hay diferentes estudios que plantean distintas visiones sobre este par. Algunos autores han sostenido la asociación baja autoestima y agresividad(31,32,) sin embargo, otros autores los ponen en cuestión debido a que las personas con alta autoestima tendían a agruparse tanto en el extremo hostil como en el no hostil(33.) Sin embargo, un aspecto importante en estas discusiones radica en que si bien, hay personas que poseen baja autoestima actúan como si no fuera así, pero muestran alta agresividad(34.) La comprensión de este par es posible a partir de pensar las lógicas de afrontamiento que se dan a partir de una necesidad de enmascarar la baja estima y responder de manera agresiva frente a la sensación de falta de recursos para hacerlo. Por lo que respondería a modalidades de respuesta agresiva relacionadas con baja autoestima, aspecto que sugerimos se siga indagando en próximos estudios de la técnica. La agresividad presenta alta frecuencia epidemiológica de morbi-mortalidad por lo que este par insta a su vez a revisar lógicas diagnósticas y aquellas vinculadas con el abordaje de la agresividad. El otro par encontrado es inseguridad/dependencia, el cual une los aspectos presentados en la versión original del CESIM, en una sola categoría. La categoríacomprende sobre todo aspectos instrumentales, no así emocionales aunque los implica. Si bien existen autores que incluyen la inseguridad como un aspecto de la dependencia35, comprendemos que ambas características se presentan articuladas. Un aspecto a destacar es que la bibliografía sobre dependencia generalmente se refiere a las mujeres como principal persona dependiente, haciendo hincapié en dependencias sostenidas desde el modelo machista heteropatriarcal,aunque no lo expliciten.Hay investigaciones que sostienen esto pero indican que la dependencia es de ambos sexos sólo que en mujeres y en varones se encuentran patrones cognitivos diferentes36. Así la categoría queda conformada por aspectos del orden cognitivo como actitudinal y emocional, relacionándose según la puntuación con una afectación en autonomía en la vida cotidiana relacionada con la baja autoestima y las dudas frente la toma de decisiones como al emprender acciones buscando apoyo social para hacerlo.

Otro dato de interés refiere a que los reactivos de enfermedades psicosomáticas se agruparon en distintos factores no interpretables desde la teoría, replicando estos hallazgos lo informado en estudio previos 1. A pesar de esta dificultad, los autores del CESIM consideran de importancia incluir los indicadores de enfermedades psicosomáticas dentro del constructo de síntomas afectivos1. En consecuencia, en el presente estudio se decidió obtener una medida global de sintomatología mentalque incluyera estos reactivos. Dicha medida presentó adecuadas propiedades psicométricas y fue seleccionada para describir los niveles de sintomatología mental en la población en estudio. Esta decisión se fundamenta en el hecho de que los síntomas psicosomáticos son parte de las categorías nosológicas psiquiátricas(28) y se entienden como manifestaciones de malestar psíquico (29) que presentan una alta correlación con situaciones estresantes que no son afrontadas adecuadamente(30.) Además, en estudios epidemiológicos dichos síntomas son de relevancia para el diagnóstico, debido a que presentan una alta prevalencia en los sectores socialmente vulnerables(9.)

En relación con el análisis descriptivo en función de variables sociodemográficas: edad, sexo, nivel de educación, estado civil y región geográfica se observan coincidencias de los resultados obtenidoscon estudios previos $(3,18,27$.) Los datos de carga de sintomatología según sexo coinciden con los hallazgos del uso de esta técnica en población general $(3,14,18,27$.

Un análisis especial entendemos que merece la sintomatología hallada según el sexo. En el presente estudio se observa mayor puntuación sintomatológica en mujeres que en varones. Los estudios epidemiológicos indagados coinciden con esta característica $(3,14,18,27$, aspecto que se repite en los datos a nivel mundial10. Este punto hace que se genere la 
pregunta sobre la posibilidad de la existencia de sesgo sexo-genéricos frente a este aspecto. Investigaciones sobre salud mental y género muestran que los factores sociales establecen diferencias injustas entre sexos, tanto roles asignados como estereotipos dominantes de femeneidad y masculinidad(37.) Así, alleer las asociaciones de sintomatología mental desde la perspectiva de género y analizando que la información viene desde el auto reporte, nos permiten pensar que el mayor registro emocional y conciencia sobre el propio cuerpo que tienen las mujeres puede repercutir en el reconocimiento sintomático; mientras que en los varones la necesidad de ser funcionales, fuertes y productivos, podría asociarse a poca virilidad(38.)

En relación a la sintomatología mental y los niveles educativos los resultados coinciden con los estudios nacionales e internacionales, menor nivel educativo se relaciona con tasas más altas de sintomatología $(3,14,38,39$.) Por lo que se deduce que las herramientas dadas por la educación formal afectan los procesos de salud-enfermedad en las personas.

Las edades comprendidas entre los 18 y 34 años de edad se asociaron con cualquier trastorno y con cada clase de trastorno evaluada $(3,10,18,27$.) Este aspecto puede relacionarse con el monto de estrés presente en las edades en donde se supone el ingreso a la etapa productiva capitalista de las personas y que a su vez se asocian con la salida del hogar de origen sea de manera material o simbólica con ingresos o composición de nuevas redes vinculares.

La mayor presencia de sintomatología en Mendoza puede ser comprendida por varios factores contextuales. Por un lado, el modo productivo característico de la zona se asocia a gran presencia de trabajo precarizado como por ejemplo, la venta ambulante, construcción, entre otros; además, la región en su conformación poblacional posee altas tasas de personas migrantes, así como personas que han sido desplazadas por el impacto de desastres naturales o con dificultades habitacionales(14,38) En la provincia de Buenos Aires, la mayor prevalencia de malestar psicológico en la población de Avellaneda en comparación con La Plata, puede relacionarse con el hecho de que en Avellaneda gran parte de la actividad laboral se desarrollaba a través del tren, cuando este fue cerrado y el aparato industrial debilitado y achicado, se piensa que impactó notablemente en la saludde los adultos y este suceso se relacionó con mayor sintomatología mental(18.) Sin embargo, es necesario tener mejores instrumentos para indagar las condiciones de inserción social e histórica de las personas a fin de conocer si éstos son los factores que inciden sobre la sintomatología mental.

\section{Conclusiones}

Poseer instrumentos con validez ecológica y adecuadas propiedades psicométricas es un aspecto de relevancia para pensar en contexto los procesos de salud-enfermedadatención.A esto se hace inminente incorporar una lectura con enfoque social, teniendo en cuenta la complejidad y las multideterminaciones de las condiciones sociales.

El CESIM es un instrumento de tamizaje diseñado en Argentina para valorar sintomatología mental. Los estudios iniciales del CESIM identificaron una estructura factorial compleja, señalando la importancia de emplear una versión abreviada. El presente trabajobrinda evidencia sobrelas propiedades psicométricas de esta versión e indica que el CESIM es un instrumento válido y confiable para valorar sintomatología mental en adultos argentinos. A la vez, los factores hallados sugieren categorías diagnósticas que no se adecuan exactamente a los cuadros nosológicos propuestos por el DSM V ni por la CIE 10, aspectos que conllevan desafíos para próximas investigaciones. Estos podrían tener como propósito conocersi nuestra sociedad posee cuadros nosológicos propios que se diferencien de los producidos desde instituciones internacionales que cuentan con la hegemonía médicopsicológica. Ya que en esta investigación el contexto estaría proponiendo cuadros sintomáticos de cualidades diferentes y por lo tanto también sentaría preguntas sobre los modos de intervención que se utilizan habitualmente y los que pueden proponerse a partir 
de estas categorías.

Se presentan datos de referencia para población argentina, los cuales son escasos en investigaciones en población general. Por lo tanto, la investigación proporciona características epidemiológicas en población que no necesariamente ha realizado consultas en salud mental con efectores de salud. La investigación presenta como limitación que la muestra no es representativa para todo el país, por lo que se sugiere realizar nuevos estudios en otros contextos y regiones.

Agradecimientos:

Se agradece al Dr. Enrique Saforcada y equipo por la facilitación de información de las investigaciones realizadas por el mismo para realizar el presente estudio.

\section{Bibliografía}

1. Grasso L, Burijovich J. Cuestionario Epidemiológico de Sintomatología Mental (CESIM). Córdoba: Dirección General de Salud Mental de la Provincia de Córdoba; 1995.

2. Muñoz-Rodríguez M, Basco ME. Indagaciones epidemiológicas en salud mental : usos de servicios de salud y percepción del apoyo social. Rev salud pública. 2016;18(2):188-200.

3. Stagnaro JC, Cía AH, Aguilar Gaxiola S, Vázquez N, Sustas S, Benjet C, et al. Twelve-month prevalence rates of mental disorders and service use in the Argentinean Study of Mental Health Epidemiology. Soc Psychiatry Psychiatr Epidemiol [Internet]. 2018;53(2):121-9. Disponible en: http://dx.doi.org/10.1007/ s00127-017-1475-9

4. Almeida Filho N, Rouquayrol M. Introducción a la epidemiología. Buenos Aires: Lugar; 2011.

5. De Lellis M. Perspectivas en Salud Pública y Salud Mental. Buenos Aires: Nuevos tiempos; 2015.

6. Morales Calatayud F. Introducción a la Psicología de la Salud: un enfoque integral. Buenos Aires: Nuevos tiempos; 2016.

7. Organizacion Panamericana de Salud. Informe sobre los sistemas de salud mental en America Latina y el Caribe [Internet]. Washington: OMS; 2013. Disponible en: http://www.paho.org/per/images/stories/FtPage/2013/WHO-AIMS.pdf

8. IHMD measuring what maters (2018). [online] Available at: http://www.healthdata. org/ argentina [Accessed 21 oct. 2018].

9. Rodríguez JJ, Kohn R, Aguilar-Gaxiola S.editores. Epidemiología de los trastornos mentales en América Latina y el Caribe.Washington: OPS; 2009.

10. Kohn R, Levav I, Miguel J, Almeida C De, Vicente B, Andrade L, et al. Los trastornos mentales en América Latina y el Caribe : asunto prioritario para la salud pública. Rev Panam Salud Pública [Internet]. 2005;18(401):229-40. Disponible en: http://www.scielosp.org/pdf/rpsp/v18n4-5/28084.pdf

11. Massa M. Plan Nacional de Salud Mental. Buenos Aires: Ministerio de Salud; 2013.

12. Casullo M, Pérez M. El inventario de síntomas SCL-90-R de L Derogatis. Adaptión UBA CONICET [Internet]. 2008;1-8. Disponible en: http://www.psi. uba.ar/academica/carrerasdegrado/psicologia/sitios_catedras/obligatorias/070_ psicoterapias $1 /$ material/inventario_sintomas.pdf

13. Brenlla ME, Aranguren M. Adaptación de la Escala de Malestar Psicolólogico de Kessler (K10). Rev Psicol la Pontif Univ Católica del Perú. 2010;28(2):310-40.

14. Basco ME. Estudio epidemiológico de sintomatología mental en adultos del barrio Lihue de Guaymallén. Universidad Nacional de Cuyo; 2017.

15. Janca A, Chandrashekar CR. Composite international diagnostic interview (CIDI) 
researchers manual. [Internet]. Switzerland: World Health Organization. Division of Mental Health; 1995. Disponible en: http://apps.who.int//iris/handle/10665/62036

16. First MB, Williams J, Karg R, Spitzer. Structured Clinical Interview for DSM-5Research Version (SCID-5 for DSM-5, Research Version; SCID-5-RV). Arlington: American Psychiatric Association.; 2015.

17. Bertelsen A, Vazquez-Vaquero JL, Brugha TS, Chatterji S, Compton WM, Göñu A, et al. Cuestionarios para la Evaluación Clínica en Neuropsiquiatría. Washington: OMS; 1995.

18. Saforcada E, De Lellis M, Martínez Calle S. Estudio epidemiológico comparativo de sintomatología de salud mental en adultos del Partido de Avellaneda y la Ciudad de La Plata , 2011. Anu Investig [Internet]. 2012;19:141-51. Disponible en: http:// www.redalyc.org/articulo.oa?id=369139948014

19. Muñoz Rodríguez M, Basco ME, Torres ME, Mosconi R, Casciani C, Videla I, et al. Las redes comunitarias como factor interviniente en la salud mental desde un estudio epidemiológico. En: Straniero C, Tosi C, Luna M, editores. Psicología y compromiso social. Buenos Aires: Federación de Psicólogos de la República Argentina; 2016. p. 366-77.

20. Rivera L De, Rodríguez-Pulido F, Sierra López A. El método epidemiológico en salud mental. Barcelona: Masson Salvat; 1993.

21. Burijovich J, Sandomiraky M. Los desempleados: Malestar psicológico, apoyo social e intervención estatal . Rev Adm Pública y Soc. 1998;11:103-14.

22. Conci M. El malestar psicológico en docentes de la ciudad de Córdoba. Universidad Empresarial Siglo XXI; 2009.

23. Furlan LA, Ferrero MJ, Gallart G. Ansiedad ante los Exámenes, Procrastinación y Síntomas Mentales en Estudiantes Universitarios [Internet]. Vol. 6, Revista Argentina de Ciencias del Comportamiento. 2014: 31-9. Disponible en: revistas. unc.edu.ar/index.php/racc

24. Rebuffo M, Siravegna MS, Medrano LA. Comorbilidad depresiva en mujeres con trastornos de conducta alimentaria. Psicol y Salud [Internet]. 2013;22(2):215-24. Disponible en: http://revistas.uv.mx/index.php/psicysalud/article/view/546

25. León O, Montero I. Métodos de investigación en psicología y educación. Madrid: McGraw Hill; 2007.

26. Censos IN de E y. Datos sociodemográficos [Internet]. Argentina; 2001. Disponible en: https://www.indec.gob.ar/micro_sitios/webcenso/censo2001s2/ampliada index.asp?mode $=01$

27. Burijovich J, D'Lucca A, Berra C. Investigación en salud mental: de los cordobeces y sus malestares. Córdoba: Sub-dirección General de Salud Mental del Gobierno de la Provincia.; 1999.

28. López J, Amparo SY. La somatización como síntoma y como síndrome: una revisión del trastorno de somatización. Rev Psicopatología y Psicol Clínica. 2002;7(2):7393.

29. Holloway KL, Zerbe KJ. Simplified approach to somatization disorder: When less may prove to be more. Postgrad Med. 2000;108(6).

30. Wimbush F, Nelson M. Handbook of Stress , Coping, and Health. En: Hill V, editor. London: Sage; 2000.

31. Gondolf E. Changing men who batter: A developmental model for integrated interventions. Journal of family violence, 1985; 2(4):335-345. https://doi. org/10.1007/BF00993299

32. Donnellan MB, Trzesniewski KH, Robins RW, Moffitt TE, Caspi A. Low Self-Esteem Is Related to Aggression, Antisocial Behavior, and Delinquency. Psychological Science. 2005; 16(4):328-335 DOI:10.1111/j.0956-7976.2005.01535.x

33. Kernis M, Grannemann B, Barclay L. Stability and level of self-esteem as predictors 
of anger arousal and hostility. Journal of Personality and Social Psychology. 1989;56:1013-1022.

34. Olweus D. Bullying at school: Long-term outcomes for the victims and an effective school-based intervention program. In R. Huesmann (Ed.), Aggressive behavior: Current perspectives: 97-130. New York: Plenum Press; 1994.

35. Castelló J. Dependencia emocional. Características y tratamiento. Madrid: Alianza; 2005.

36. Lemos M, Londoño NH. Construcción y validación del cuestionario de dependencia emocional en población colombiana. Acta Colomb Psicol [Internet]. 2006;9(2):127-40. Disponible en: http:// dialnet.unirioja.es/servlet/articulo? codigo $=2263254$ \&info $=$ resumen\&idioma $=$ SPA

37. Linardelli F. Salud mental y género. Diálogos y contrapuntos entre biomedicina, feminismos e interseccionalidad. MILLCAYAC - Rev Digit Ciencias Soc. 2015;2(3):199-224.

38. Muñoz Rodríguez M. Estudio epidemiológico de sintomatología mental desde una aproximación integral a los procesos de salud-enfermedad en personas adultas de un distrito urbano de Guaymallén-Mendoza.Universidad Nacional de Cuyo. 2018. Sin defender. 